\title{
The Impacts of Future Time Perspective on the Academic Delay of Gratification among Chinese and Thai University Students
}

\author{
Chao-Hua Fang and Ren-Cheng Zhang \\ Dhurakij Pundit University \\ Bangkok, Thailand
}

\begin{abstract}
This study explored the relationship between future time perspectives and academic delay of gratification among university students in China and Thailand. Specifically, a cross-cultural perspective was employed to establish a structural equation model between the two variables. Surveys were distributed to 200 randomly selected students from five universities in China and Thailand. All resulting data were sorted using SPSS 22.0, while statistical analyses were conducted using the AMOS 17.0 software. Results showed that future time perspectives had a significant impact on the academic delay of gratification among Chinese university students, but there was no significant influence among Thai university students. Future time perspectives and academic delay of gratification among university students are highly relevant topics in the field of development and educational psychology. The following article discusses the practical significance of these results in regard to mental health education. These findings should also provide a valuable point of reference in the general pedagogical context. Therefore, it provides evidence for improving the competitiveness of future college students and cultivating first-class international talents, in order to play the value and role of application in university education.
\end{abstract}

Keywords: future time perspectives; academic; delay of gratification; Chinese university students; Thai university students.

\section{Introduction}

Human nature is future-oriented due to a yearning for the pursuit of human development; it is full of infinite power and creativity (Simons, Vansteenkiste, Lens, \& Lacante, 2004). However, people are different in perceiving future time, understanding the value of future time, managing future time, and decision making. That is, people have different future time perspective (Husman, McCann, \& Crowson, 2000). Our understanding of time is also related to individual development (Liu, 2012). In this context, different perceptions of future time are development-oriented. For example, many university students 
are now looking forward to exploring the future. It is thus essential to comprehensively study the development of individual future time perspectives in this setting context. Here, academic delay of gratification refers to how students make choices regarding their expectations and aims during the learning process. That is, they decide how to begin pursuing long-term academic performance and may choose to give up immediate gratification and, or other rewards in favor of self-control (Bembenutty \& Karabenick 1998). Research into the academic delay of gratification is relatively new, but has proven to be helpful in understanding and explaining student behaviors in the learning context. Because academic performance is one of the most essential things in the lives of university students, it is necessary to study the academic delay of gratification among their demographic. The research results of a questionnaire study by Zhuang (2011) showed that future time perspectives and academic delay of gratification were significantly correlated among university students. However, few studies have comprehensively focused on the relationship between future time perspectives and academic delay of gratification (Zhuang, 2011; Liu, 2012; Zhang, 2015). Further, studies isolating the postponement of gratification have tended to focus on children (Mauro \& Harris 2000). Although the current research scope now includes adolescents and adults, the areas of investigation are limited to career and consumer psychology contexts. As such, there is no complete framework for examining other issues. This study addressed this research gap by focusing on the above points in the context of two national university systems. First was Thailand, where learning culture is deeply influenced by Buddhism. The second was China, which provided a useful point of comparison due to its markedly different learning culture. This study, therefore, empirically analyzed how future time perspectives influenced academic delay of gratification among both Chinese and Thai university students to develop suggestions for related interventions.

\section{Literature review}

Future time perspectives

Research on future time perspectives first began with Wallace (1956), who defined the concept as "anthropomorphized time and a sequence of future events." Gjesme (1983) later asserted that future time perspectives entailed the general psychological abilities of anticipation, clarification, and next construction. The same study defined future time perspectives as comprising the skills of prediction, interpretation, and next development to form a type of searchlight for illuminating upcoming situations to help individuals take appropriate actions. Soon after, a study by Lens (1975) made pioneering developments in the research of future time perspectives by indicating that the concept itself was aim-oriented and could help predict behavioral consequences. This involves both the process of individual development and the idea of individually based internal stability.

Scholars tend to regard the ability to use future time perspectives as stable, thus guiding individual future aims (Van Calster, Lens, \& Nuttin, 1987). Simons et al. (2004) literature review indicated that future time perspectives were seen as a combination of cognition, experiences, and behaviors related to individual 
cognitive structures rather than mere personality traits. Some researchers believe that future time perspectives entail personal views of time toward particular aims (Husman \& Lens 1999; Peetsma, 2000). Indeed, Kauffman and Husman (2004) asserted that the concept of future time perspective was based on how individuals anticipated and planned for the future and thus guided current actions through future thinking.

Bembenutty and Karabenick (2004) believed that the future time perspective referred to a hopeful belief in one's future aims. Here, learners with stronger feelings have a greater awareness of how current inputs influence future intentions. These individuals take existing investments and efforts as essential ways to achieve goals and can thus focus on future achievements rather than immediate satisfaction. The future time perspective is a cognitive construct. Integrating an individual's view of future time must involve the integration of the cognitive level(Ustun, 2018).

Scholars studying individual time perspective from a cognitive viewpoint have divided it into three orientations (i.e., past, present, and future) (Zimmerman, 1998). As such, these researchers believe that individual attitudes toward time consists of history, present, and future cognition (Zimbardo \& Boyd 2015). Here, individuals can use all three-time orientations to code, store, and form new expectations, aims, and opinions about situational developments (Husman, Brem, Banegas, Duchrow, \& Haque, 2015).

In sum, this study adopted the definition of future time perspective proposed by Kauffman and Husman (2004), which refers to how individuals anticipate and plan for the future while guiding current actions through future thinking.

Academic delay of gratification

Many researchers have indirectly defined the concept of academic delay of gratification. That is, it refers to the manifestation of psychological maturity in which individuals forego instant rewards for more valuable long-term results through self-control and patience (Ho, Tong, \& Jia, 2016). Academic delay of gratification is an extension of the more basic academic concept "delay of gratification." It refers explicitly to delays of gratification in the context of learning scenarios and the embodiment of individual regulatory abilities in the academic field (Watson \& Milfont 2017). Mischel and Ebbesen (1970) defined delay of gratification as a manifestation of psychological maturity in which the individual foregoes immediate gratification to achieve more valuable long-term goals through self-control and patience. Ward, Perry, Woltz, and Doolin (1989) demonstrated individual differences in applying a delay of gratification under different circumstances, satisfaction-based features, characteristics, and connotations. For example, academic delay of gratification refers to one's ability to make choices about their aims and expectations in terms of different academic tasks (e.g., preceding small rewards to pursue more valuable long-term educational purposes through self-control). Bembenutty and Karabenick (1998) originally proposed the concept of academic delay of gratification as explicitly referring to the student tendency to forego immediate gratification to pursue 
more valuable long-term aims and expectations in the context of education. Mauro and Harris (2000) proposed the term Externally Imposed Delay (EID), which entails that individuals must make choices more often when under external pressures (e.g., from parents, teachers, rules, and expectations) in real-life situations. Such externally imposed delays focus more on outside influences. Bembenutty and Karabenick (1998) found that academic delay of gratification was an essential part of the student self-control system and that resulting behavior was successful resources and control strategies. There has also been researched on the academic delay of gratification among American university students according to the academic delay of gratification scale (ADOGS) (Bembenutty \& Karabenick 1998). Here, results showed that academic delay of gratification was a component of the student self-control system involving resource-control strategies. Academic delay of gratification is an individual's self-control ability, which affects the individual's goal orientation(Shimoni, Berger, \& Eyal, 2019).

Delay of gratification is based on Mischel and Ebbesen (1970) concept of delay of satisfaction, which is described as a deliberate focus on future rewards. Ward, Perry, Woltz, and Doolin (1989) later described the idea as the ability to postpone smaller, more immediate rewards to achieve more valuable long-term goals that require patience and, or effort. As such, delay of gratification can be seen as a self-regulatory ability oriented toward future goals. Bembenutty and Karabenick (1998) emphasized it as one of the most critical components of self-control. The use of the term in the academic context is still based on Mischel and Ebbesen (1970) earlier concept. Here, academic delay of gratification occurs in learning situations as an extension of the more basic usage but focuses on explaining student learning behaviors and psychological processes. Following Bembenutty and Karabenick (1998), this study thus defined academic delay of gratification as "the tendency of university students to postpone opportunities of immediate gratification to pursue more valuable long-term learning aims in the learning situation."

Studies on the relationship between future time perspective and academic delay of gratification

Future time perspectives significantly impact academic delay of gratification. The rewards of achieving present tasks are secondary to realizing future goals for students who employ such views. Lomranz, Shmotkin, and Katznelson (1983) argue that the relationship between future time perspective and delay of gratification was based on the assumption that a lack of the ability to delay rewards damaged one's ability to anticipate and plan for the future (i.e., the essential elements involved in future time perspectives). Individuals who cannot delay gratification may thus be expected to have shorter and less-structured future time perspectives. Research has also shown that there are significant relationships between future time perspectives, delay of gratification, and social class. Here, individuals in the middle classes tend to have stronger future time perspectives and higher abilities to delay gratification than people in the lower levels. 
Meanwhile, academic delay of gratification is conducive to individuals who wish to realize future goals. Bembenutty and Karabenick (2004) studied the difference between students with future time perspectives and those without in the contexts of self-adjustment, future time perspectives, and delay of gratification. Results indicated that students chose to postpone gratification because they could perceive that it would bring value and enhance their ability to achieve long-term goals. To some extent, this ability can be attributed to will. That is individuals who decide to delay gratification willfully adopt a series of cognition-based resource-management strategies to realize long-term goals. As such, students with long-term goals tend to have specific plans for achieving them and therefore possess higher degrees of instrumental consciousness.

Bembenutty and Karabenick (2004) developed the ADOGS to evaluate the relationship between the delay of gratification and self-adjustment specifically. Their research found that students who were able to delay their gratification also used more efficient cognitive and behavioral-adjustment strategies. Further, Miller and Brickman (2004) asserted the importance of having valuable future aims; such an approach makes school tasks more meaningful, thus enhancing both individual instrumental and motivational value. In turn, this guides self-adjustment behaviors. Future intentions, therefore, play pivotal roles. Zhuang (2011) study on the relationship between future time perspectives and academic delay of gratification showed a significant and positive correlation between the two concepts among university students. The researcher further asserted that future time perspectives consisted of four factors (i.e., behavioral commitment, future effectiveness, objective consciousness, and next image). Combined, these can be used to predict academic delay of gratification among university students. Indeed, the future time perspective is closely related to the delay of gratification. That is, higher amounts of future time perspective increase the likelihood that an individual will delay gratification to achieve long-term goals. This also has a significant impact on self-realization.

However, the formation of a future time perspective is influenced by many factors, including gender, cultural background, the family environment, and education (Zhao, 2008). Thailand is a Buddhist country. However, Thai learning culture is deeply influenced by both Buddhism and Western ideas (Wen, 2012). To achieve a more thorough understanding, it is thus necessary to study the relationship between future time perspectives and academic delay of gratification in different cultural contexts. This study, therefore, examined both concepts among Chinese and Thai university students. The following hypotheses were first developed:

H1: Future time perspectives impact academic delay of gratification among Chinese university students.

H2: Future time perspectives impact academic delay of gratification among Thai university students.

According to the above Introduction, through literature review and analysis, as the basis of the research design and research structure of this research, and using 
questionnaires to collect data, in-depth discussion of the path relationship between variables.

\section{Research methods}

This study employed a survey-based approach. Following the research aims, motivations, literature review, and analyses outlined above, all resulting data were analyzed by setting a future time perspective as an independent variable and delay of gratification as a dependent variable to further explore their relationship. The specific research framework is shown in Figure 1.



Figure 1: Research framework

\section{Research instruments}

This study distributed questionnaires consisting of three parts (i.e., primary data, a future time perspective scale, and the ADOGS). Both preexisting scales were each translated into Chinese and Thai. Two experts translated the Chinese versions with high English proficiency. However, this study's researcher further modified their translation. Two other experts with high Thai skills then translated the Chinese survey into Thai. Finally, both scales received cross-cultural adjustments based on expert consultation.

The future time perspective scale

This study used the scale developed by Husman and Shell (2008), which contained 24 items across four subscales (i.e., six items each for value, connectedness, extensibility, and haste). Each item was scored using Richter's 5-point scale (i.e., 1 = "very inconsistent," 2 = "roughly inconsistent," $3=$ "ordinary," 4 = "roughly consistent," and 5 = "very consistent"). Cronbach's a coefficients for value, connectedness, extensibility, and haste were $0.77,0.75,0.80$, and 0.72 for the Chinese version, respectively, and $0.80,0.83,0.78$, and 0.85 for the Thai version, respectively. Internal consistency was determined as sufficient.

The academic Delay of Gratification scale

This study adopted Bembenutty and Karabenick (1998) the academic Delay of Gratification scale to measure the academic delay of gratification. The scale consisted of 10 total questions, each of which required students to choose one of two options that were then answered on a 4-point Likert scale (i.e., $1=$ choice A is positive, 2 = choice A is possible, $3=$ choice B is possible, and $4=$ choice $B$ is positive). Cronbach's a coefficients for the Chinese and Thai scale versions were 
0.74 and 0.95 , respectively.

\section{Research samples}

This study adopted a quantitative method to analyze data from Thai and Chinese university students. According to Thompson (2000), a formal sample analysis requires at least 200 samples. As there are 33.83 million Chinese university students, a sample size of 200 achieves a confidence error of $5.54 \%$ according to a $95 \%$ CI. On the other hand, there are 1.9 million Thai university students. As such, a sample size of 200 achieves a confidence error of 5.54\% according to a $95 \%$ CI. A convenient sampling method was thus adopted to randomly select 200 samples from universities in both China and Thailand.

\section{Implementation of the scales}

This study was divided into two stages (i.e., preliminary and formal tests). First, a pretest sampling plan entailed that colleagues of the researcher distributed the scales among their university classes. Test papers were then completed on-site (all answers were given anonymously). The experimenters first explained the study purpose, specific measurement methods, and provided testing instructions. Each scale took approximately 20 minutes to complete; all test papers were then collected and analyzed to improve validity and recovery rates. Inappropriate items were deleted to result in a formal scale for use in stage two. The researcher then contacted friends to assist in distributing the formal questionnaires according to a sampling plan. All completed questionnaires were then collected for statistical data analysis. This process resulted in 189 and 193 valid surveys from China and Thailand, respectively (invalid samples with missing and, or suspected random answers were excluded for effective rates of $94.5 \%$ and $96.5 \%$, respectively). The SPSS 22 and AMOS.17 software programs were used for all statistical analyses. A structural equation model was also constructed to examine the relationships between future time perspective and academic delay of gratification in both countries.

\section{Study results}

Structural equation model for the relationship between future time perspective and academic delay of gratification (Chinese universities)

This study examined whether future time perspective and delay of gratification were correlated according to a structural model. Specifically, this involved analyzing the degree of adaptation between the potential variable path and empirical data on both future time perspective and academic delay of gratification among Chinese university students. As shown in Figure 2, the chi-square value of model's the overall adaptation degree was $235.62(\mathrm{p}<.000)$, while the degree of freedom was 125, and the chi-square freedom ratio was 1.89 $($ RMESA $=0.07$, PGFI $=0.65$, PNFI $=0.66$, PCFI $=0.73)$; all achieved the adaptation standard. Other adaptation degree indexes also met or were close to the adaptation standard. Overall, the model was considered sufficient for studying the sample data. 


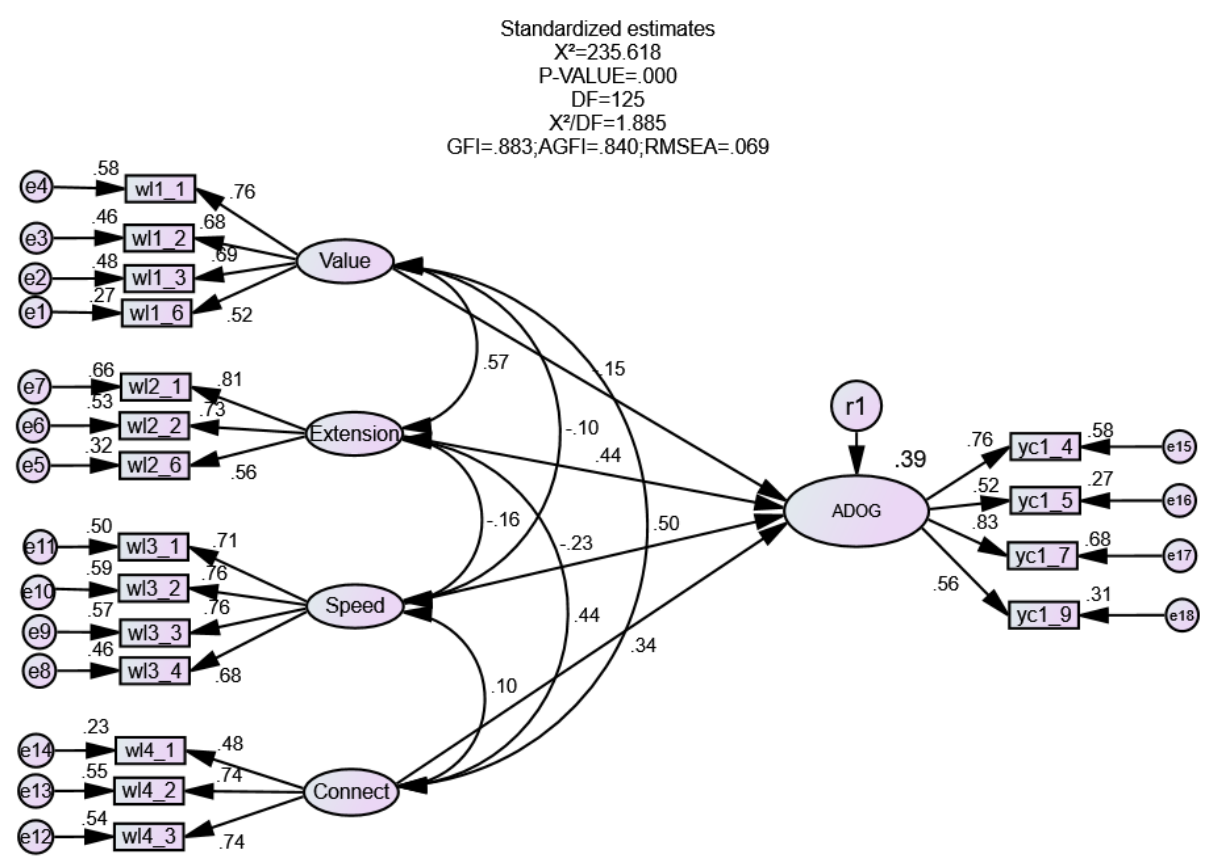

Figure 2: Structural equation model for studying the relationship between future time perspective and academic delay of gratification among Chinese university students

A model parameter estimation summary can be seen in Table 1 . The estimated values of variance were all positive, thus indicating that no unreasonable solution appeared in the model estimation. According to the observation of variable factors of load (standardized path coefficient), the composite reliability of every potential variable and value of the average variance of the amount taken could be estimated respectively (i.e., value $(\mathrm{AVE}=0.45, \mathrm{CR}=0.76$ ), extensibility $(\mathrm{AVE}=0.50, \mathrm{CR}=0.75)$, haste $(\mathrm{AVE}=0.53, \mathrm{CR}=0.82)$, connectivity $(\mathrm{AVE}=0.44, \mathrm{CR}=0.70)$, and academic delay of gratification $(\mathrm{AVE}=0.46, \mathrm{CR}=$ $0.77)$ ). This indicated that the observed variables were well-explained by the structural variables. That is, the observed variables converged with particular structural variables. The above data analysis shows that the causal relationship influenced by potential variables in the structural model conformed to the theoretical construct.

The SEM analysis results revealed no significant relationship between the value dimension of future time perspective and academic delay of gratification ( $\beta=$ $0.15, \mathrm{p}=0.212$ ) among Chinese university students. However, there were significant differences between academic delay of gratification and other dimensions, including extensibility $(\beta=0.44, p=0.001)$, haste $(\beta=0.23, p=0.009)$, and connectivity $(\beta=2.86, p=0.004)$. As such, extensibility and connectivity both had positive impacts on the academic delay of gratification, while haste had a negative impact. 
Table 1: Model parameter estimation summary on the impact path of future time perspective on the academic delay of gratification among Chinese university students

\begin{tabular}{cccc}
\hline & S.E. & C.R. & $\begin{array}{c}\text { Standardized } \\
\text { Regression } \\
\text { Weights }\end{array}$ \\
\hline Value $\rightarrow$ Academic Delay of Gratification & 0.19 & -1.25 & -0.15 \\
Extension $\rightarrow$ Academic Delay of Gratification & 0.17 & $3.41^{* * *}$ & 0.44 \\
$\quad$ Speed $\rightarrow$ Academic Delay of Gratification & 0.09 & $-2.62^{* *}$ & -0.23 \\
Connection $\rightarrow$ Academic Delay of Gratification & 0.12 & $2.86^{* *}$ & 2.86 \\
\hline
\end{tabular}

${ }^{* *} \mathrm{p}<.01 .{ }^{* * *} \mathrm{p}<.001$

Structural equation model analysis of future time perspective and academic delay of gratification among Thai university students

This study examined whether future time perspective and delay of gratification were correlated and established a structural equation model of the relationship between the two variables to analyze the adaptation degree between the potential variable path and empirical data among Thai university students. As shown in Figure 3, the chi-square value of the overall adaptation degree of the model was $615.70(\mathrm{p}<.000)$, while the degree of freedom was 367, and the chi-square degree of freedom was 1.68 (RMR=0.04, RMSEA=0.06, IFI=0.92, $\mathrm{TLI}=0.91, \mathrm{CFI}=0.92$, PGFI=0.70, PNFI=0.73, PCFI=0.83). As such, all factors met the adaptation standard; other adaptation degree indexes also meet or were close to the adaptation standard. Overall, the model was well-adapted to the sample data.

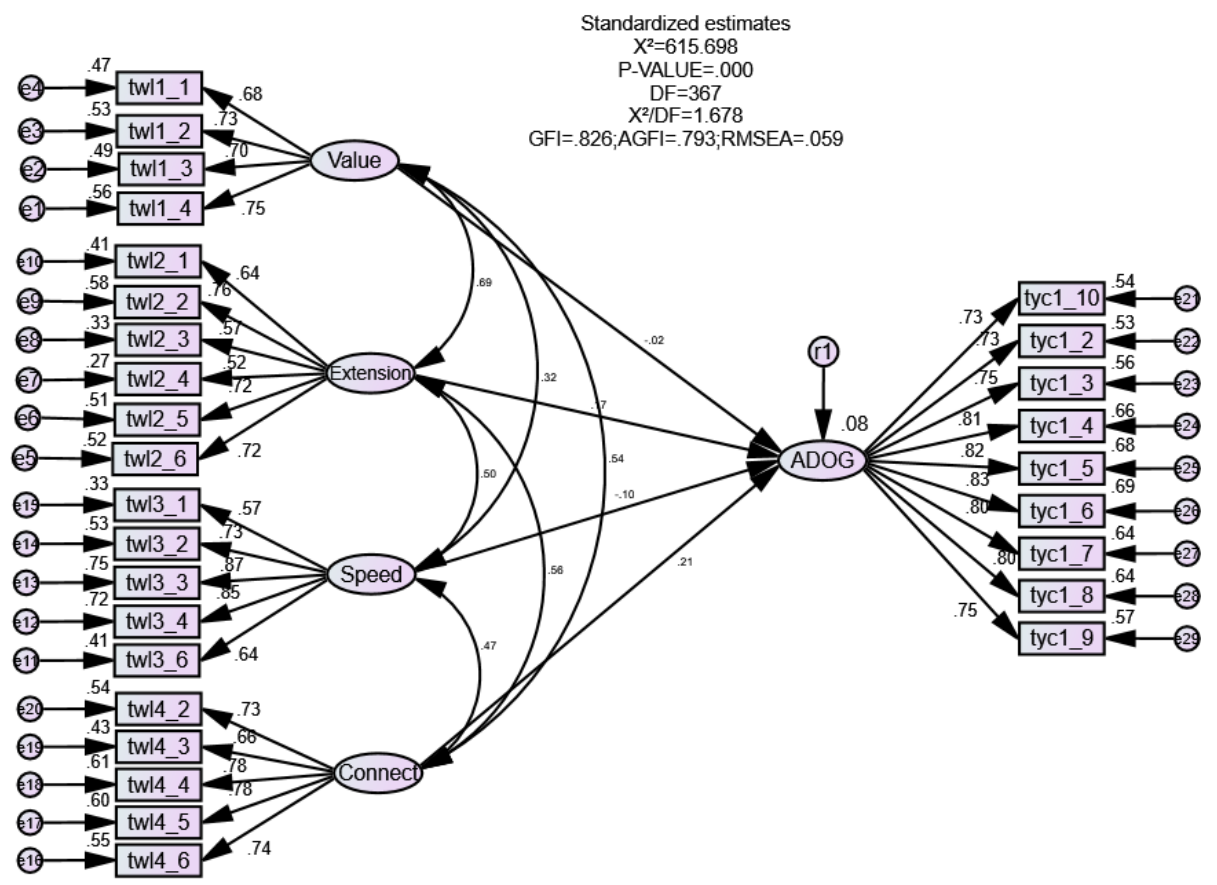

Figure 3: Structural equation model for studying the relationship between future time perspective and academic delay of gratification among Thai university students 
A model parameter estimation summary can be seen in Table 1.2. The estimated costs of variance were all positive, thus indicating that no absurd solution appeared in the model estimation. According to the observation of variable factors of load (standardized path coefficient), the composite reliability of every potential variable and value of the average variance of the amount taken could be estimated respectively (i.e., value $(\mathrm{AVE}=0.51, \mathrm{CR}=0.81)$, extensibility $(\mathrm{AVE}=0.44, \mathrm{CR}=0.82)$, haste $(\mathrm{AVE}=0.55, \mathrm{CR}=0.86)$, connectivity ( $\mathrm{AVE}=0.55$, $\mathrm{CR}=0.86$ ), and academic delay of gratification (AVE=0.61, $\mathrm{CR}=0.93)$ ), thus indicating that the observed variables of the measurement model were well-explained by the structure variables. That is, the observed variables converged with particular structural variables. The above data analysis shows that the causal relationship influenced by potential variables in the structure model conformed to the theoretical construct.

The SEM analysis results indicated no significant relationship between the dimensions of value $(\beta=-0.02, p=0.857)$, extensibility $(\beta=0.17, p=0.238)$, haste $(\beta=-0.10, p=0.301)$, connectivity $(\beta=0.21, p=0.055)$, and academic delay of gratification. That is, none of the future time perspective dimensions had significant impacts on the academic delay of gratification among Thai university students. This may be due to the specific Buddhist values held by Thai university students (Root, 2016).

Table 2: Model parameter estimation summary of the impact path of future time perspective on the academic delay of gratification among Thai university students

\begin{tabular}{cccc}
\hline & S.E. & C.R. & $\begin{array}{c}\text { Standardized } \\
\text { Regression } \\
\text { Weights }\end{array}$ \\
\hline Value $\rightarrow$ Academic Delay of Gratification & 0.17 & -0.18 & -0.02 \\
Extension $\rightarrow$ Academic Delay of Gratification & 0.19 & 1.18 & 0.17 \\
$\quad$ Speed $\rightarrow$ Academic Delay of Gratification & 0.15 & -1.03 & -0.10 \\
Connection $\rightarrow$ Academic Delay of Gratification & 0.15 & 1.92 & 0.21 \\
\hline
\end{tabular}

Through the above construction of the structural equation model for the future time perspective and academic delay of gratification among Chinese and Thai students, it shows that the future time perspective has an impact on the academic delay gratification among Chinese university students, hypotheses $\mathrm{H} 1$ was verified. The future time perspective has no effect on academic delay gratification among Thai university students, hypotheses $\mathrm{H} 2$ was not been verified.

\section{Discussion}

The above theoretical discussion and research concepts were used to create structural equation models establishing a relationship between future time perspective and academic delay of gratification among university students. A path analysis was also conducted to explore the internal relations between the two variables. There were several other significant findings. 
First, the value dimensions of future time perspective and academic delay of gratification among Chinese university students were not significantly correlated. This may be because these students felt increasingly good about the status quo, the higher they valued their scheduled time. As such, they did not pay substantial amounts of attention to delay of gratification (Zimbardo \& Boyd 2015). However, the factors of extensibility and connectivity were positively correlated with a delay of gratification. That is, students who were extensibilityand connectivity-oriented usually felt that future time was limited and thus had a clearer understanding of long-term learning goals, paid more attention to a future time, and restrained the impulse to receive immediate enjoyment to achieve long-term learning aims (Mello \& Worrell 2015). On the other hand, the dimension of haste was negatively correlated with a delay of gratification. In other words, the slower the perceptual period, the time of the student, the higher their confidence in future achievement. As such, they approach the future with full expectations and understand both the value and significance of their goals. This helps them postpone enjoyment to realize more valuable long-term learning aims (Eccles \& Wigfield 2002).

There were no significant correlations between all dimensions of future time perspective and delay of gratification among Thai university students, thereby distinguishing them from Chinese university students in this regard. This may be because Thai university students are more influenced by Buddhist values (Wen, 2012). Thai Buddhism believes that the status and destiny of human beings in this life are determined by the accumulation of karma in the previous experience. What human beings do in this life cannot change the current fate and status, only affect the next life. People who have wealth or power in this life are all caused by the extremely high "karma accumulation" in the previous experience, so the public can only identify with what these people have, and do good deeds to accumulate virtue to strive for the next life. This has resulted in the Thai people's thinking mode of being aloof from the rest of the world, being happy to know what they want, and even not seeking progress. Reflected in the learning culture of Thai college students, it has formed a view of time that attaches great importance to the future, but the study lacks hard work. At the same time, under this way of thinking, the sense of honor brought by competition and winning is not of high value. Meanwhile, the Thai educational system is relatively comprehensive and rich; vocational education is specially developed, thus providing more choices for future development while reducing the pressure to find employment (Root, 2016). As such, Thai students tend to focus on developing survival skills and personal interests rather than work or more advanced education. At the same time, the decrease mentioned above in the pressure to find employment results in less study pressure, thus leading to a lack of hard work and practice as well as undisciplined study attitudes (Pongsuwan, Boddy, Woods, \& Balthip, 2016). Therefore, although Thai college students attach importance to the future time perspective, due to the lack of employment pressure, learning pressure, and competitive awareness, the future time perspective of Thai college students have no significant impact on the academic delay of gratification. 
The above analyses and discussions on the internal relationships between the two variables have further deepened the literature on both future time perspectives (Kauffman \& Husman 2004) and academic delay of gratification (Bembenutty \& Karabenick 1998). These results can be used by government sources to design relevant macroscopic educational guidance policies to help university students establish better future time perspectives and improve their abilities to delay gratification in the academic context. The same findings should also be relevant to those working in student affairs while providing practical guidance for universities in training international students in both areas.

\section{Conclusions}

Future time perspectives and academic delay of gratification among university students comprise an essential topic in the field of development and educational psychology. This study explored the relationship between both concepts among Chinese and Thai university students to provide a basis for cross-cultural comparison. Further, structural equation models were developed for use in each university context. Results clearly showed that the future time perspective had a significant impact on the academic delay of gratification among Chinese university students. However, no such effect was found among Thai university students. These findings have important implications for mental health education in the university systems of both countries and provide valuable references for future teaching practices. While some differences are likely the result of cultural values (and other factors such as gender, socioeconomic background, and, or education level), general interventions can be implemented to improve academic focus as it relates to future goal achievement. This is also highly important in helping students achieve their current goals in the academic setting. Below is some vital suggestion for such interventions as well as advice on the direction of future research.

\section{Suggestions for further research}

This paper puts forward three significant ideas related to both practical application and future research based on the above results.

1. Enhance student awareness of the future time perspective to facilitate the establishment of positive academic aims.

On the one hand, universities should help students reorient their future visions, guide them toward future orientations, clarify appropriate academic directions, and enhance their confidence and courage in achieving goals. On the other hand, the concept of definite academic aim not only refers to positive tropism but more importantly, involves how proper academic purposes can be used to achieve future goals through current actions. Academic purposes should be established to help university students conform to the status quo while motivating their desire for achievement to effectively improve their ability to delay gratification in the academic context.

2. Provide a clear image of the future time perspective and encourage university students to establish appropriate development plans

Detailed future development plans help university students obtain brighter visions of the future, understand the necessary steps needed to reach their goals, 
and decide what specific tasks they must accomplish at each level. Such plans can also be used as reference points for effectively monitoring progress. Some universities have already begun to offer development planning courses to help students establish plans. However, professional development planning guidance centers staffed by full-time teachers can provide even better-planning consultation services to students. This will improve the overall quality of the university planning process, which will then help students delay gratification.

3. Improve student behavioral commitments to future time perspectives and guide them in developing time-management strategies and skills.

One of the most common problems encountered when enacting academic aims and plans is finding a way to give appropriate consideration to all parties while making sufficient use of limited time resources. As such, university students must be guided in obtaining efficient time-management strategies and skills (e.g., keeping task-time logs, classifying and sorting tasks, establishing completion deadlines, and implementing tasks according to strict plans). These elements will help students make scientific time decisions while improving their ability to delay gratification when conflicts arise that threaten their long-term academic aims.

\section{Research limitations and prospects}

1. This study's investigation into Chinese and Thai university students employed a convenient sampling process. However, this process could not cover all regions of China and Thailand due to limited financial and material resources. As such, there is limited representativeness. Future studies should thus consider more scientific and rigorous sampling methods to improve both representativeness and external validity.

2. This research was mostly quantitative and used the questionnaire survey as a primary way to collect data. Further, all reviews were self-reported. This process is vulnerable to bias and, or misinformation because respondents may choose their answers based on self-expectations or social pressures. Future studies should thus implement scientific interviews or experiments to obtain qualitative data and improve internal validity.

3. This study only considered the background variables of gender, university major, grade level, and nationality. Additional demographic variables should be considered in future studies (e.g., family origins and homelife practices).

\section{References}

Bembenutty, H., \& Karabenick, S.A. (1998). Academic delay of gratification. Learning and Individual Differences, 10(4), 329-346. https:// doi.org/10.1016/S1041-6080(99)80126-5

Bembenutty, H., \& Karabenick, S. A. (2004). Inherent Association Between Academic Delay of Gratification, Future Time Perspective, and Self-Regulated Learning. Educational Psychology Review, 16(1), 35-57. https://doi.org/10.1023/b:edpr.0000012344.34008.5c

Eccles, J. S., \& Wigfield, A. (2002). Motivational beliefs, values, and goals. Annual Review of Psychology, 53(1), 109-132.

https://doi.org/10.1146/annurev.psych.53.100901.135153 
Gjesme, T. (1983). On the concept of future time orientation: considerations of some functions' and measurements' implications. International Journal of Psychology, 18(1-4), 443-461. https://doi.org/10.1080/00207598308247493

Ho, S.-Y., Tong, E. M., \& Jia, L. (2016). Authentic and hubristic pride: differential effects on delay of gratification. Emotion, 16(8), 1147-1156. https://doi.org/10.1037/emo0000179

Husman, J., \& Lens, J. (1999). The role of the future in student motivation. Educational Psychologist, 34(2), 113-125. http://dx.doi.org/10.1207/s15326985ep3402_4

Husman, J., McCann, E., \& Crowson, H.M. (2000). Volitional strategies and future time perspective: embracing the complexity of dynamic interactions. International Journal of Educational Research, 33(7-8), 777-799. https://doi.org/10.1016/S0883-0355(00)00050-1

Husman, J., \& Shell, D. F. (2008). Beliefs and perceptions about the future: a measurement of future time perspective. Learning and Individual Differences, 18(2), 166-175. https:// doi.org/10.1016/j.lindif.2007.08.001

Husman, J., Brem, S. K., Banegas, S., Duchrow, D. W., \& Haque, S. (2015). Learning and future time perspective: the promise of the future-rewarding in the present. In M. Stolarski, N. Fieulaine, and W. van Beek (Eds.), Time Perspective Theory; Review, Research and Application (pp. 131-141). Cham, Germany: Springer. https://doi.org/10.1007/978-3-319-07368-2_8

Kauffman, D. F., \& Husman, J. (2004). Effects of time perspective on student motivation: introduction to a special issue. Educational Psychology Review, 16(1), 1-7. https:// doi.org/10.1023/B:EDPR.0000012342.37854.58

Lens, W. (1975). Sex differences in attitudes towards personal past, present, and future. Psychological Belgica, 15(1), 29-33.

Liu, L. (2012). Research on the relationship between the future time insight, academic delay of gratification and academic self-efficacy of junior middle school students (master's thesis). Shenyang Normal University, China.

Lomranz, J., Shmotkin, D., \& Katznelson, D. (1983). Coherence as a measure of future time perspective in children and its relationship to delay of gratification and social class. International Journal of Psychology, 18(1-4), 407-413. https://doi.org/10.1080/00207598308247490

Mauro, C. F., \& Harris, Y. R. (2000). The influence of maternal child-rearing attitudes and teaching behaviors on preschoolers' delay of gratification. The Journal of Genetic Psychology: Research and Theory on Human Development, 161(3), 292-306. http://dx.doi.org/10.1080/00221320009596712

Mello, Z. R., \& Worrell, F. C. (2015). The past, the present, and the future: a conceptual model of time perspective in adolescence. In M. Stolarski, N. Fieulaine, and W. van Beek (Eds.), Time Perspective Theory; Review, Research and Application (pp. 115-129). Cham, Germany: Springer. http:/ /dx.doi.org/10.1007/978-3-319-07368-2_7

Miller, R. B., \& Brickman, S. J. (2004). A model of future-oriented motivation and self-regulation. Educational Psychology Review, 16(1), 9-33. https://doi.org/10.1023/B:EDPR.0000012343.96370.39

Mischel, W., \& Ebbesen, E. B. (1970). Attention in delay of gratification. Journal of Personality and Social Psychology, 16(2), 329-337. https://doi.org/10.1037/h0029815

Peetsma, T. T. (2000). Future time perspective as a predictor of school investment. Scandinavian Journal of Educational Research, 44(2), 177-192. https://doi.org/10.1080/713696667

Pongsuwan, C., Boddy, J., Woods, M., \& Balthip, Q. (2016). Thai hierarchical culture influences undergraduate nursing students' learning. Jurnal Sains Sukan $\mathcal{E}$ Pendidikan Jasmani, 5(1), 9-21. 
Root, S. (2016). Understanding Thai culture: exploring the effect of academic stress in students' learning orientation. AJMI-ASEAN Journal of Management \& Innovation, $3(2), 44-58$.

Shimoni, E., Berger, A., \& Eyal, T. (2019). Priming pride promotes delay of gratification. Motivation and Emotion, 43(5), 786-802. https://doi.org/10.1007/s11031-019-09778-6

Simons, J., Vansteenkiste, M., Lens, W., \& Lacante, M. (2004). Placing motivation and future time perspective theory in a temporal perspective. Educational Psychology Review, 16(2), 121-139. https://doi.org/10.1023/B:EDPR.0000026609.94841.2f

Thompson, B. (2000). Ten commandments of structural equation modeling. In L.G. Grimm \& P. R. Yarnold (Eds.), Reading and Understanding More Multivariate Statistics (pp.261-283). Washington, US: American Psychological Association.

Ustun, U. D. (2018). Future Time Perspective and Reduction in Motivation for Recreation Department Students in Turkey. Journal of Educational Issues, 4(2), 27-35. https://doi.org/10.5296/jei.v4i2.13618

Van Calster, K., Lens, W., \& Nuttin, J. R. (1987). Affective attitude toward the personal future: impact on motivation in high school boys. The American Journal of Psychology, 100(1), 1-13. http:/ / dx.doi.org/10.2307/1422639

Wallace, M. (1956). Future time perspective in schizophrenia. The Journal of Abnormal and Social Psychology, 52(2), 240-245. http://dx.doi.org/10.1037/h0039899

Ward, W., E., Perry, T. B., Woltz, J., \& Doolin, E. (1989). Delay of gratification among Black college student leaders. Journal of Black Psychology, 15(2), 111-128. https:// doi.org/10.1177/00957984890152004

Watson, S. J., \& Milfont, T. L. (2017). A short-term longitudinal examination of the associations between self-control, delay of gratification and temporal considerations. Personality and Individual Differences, 106, 57-60. https://doi.org/10.1016/j.paid.2016.10.023

Wen, W. (2012). Survey of Thai learning culture and its impact on Chinese teaching (Master's thesis). Shandong University, China.

Zhang, Y. (2015). Study on the influence of time insight on academic procrastination of university students. Journal of Hubei Correspondence University, 28(20), 21-22.

Zhao, Z. (2008). Commentary on time insight research status quo. Journal of Tonghua Normal University, 29(3), 103-104.

Zhuang, Y. (2011). Research into the relationship between college students' future time insight and academic delay of gratification. Theory and Practice of Education, 31(1), 42-45.

Zimbardo, P. G., \& Boyd, J. N. (2015). Putting time in perspective: a valid, reliable individual-differences metric. In M. Stolarski, N. Fieulaine, and W. van Beek (Eds.), Time Perspective Theory; Review, Research and Application (pp. 17-55). Cham, Germany: Springer. https://doi.org/10.1007/978-3-319-07368-2_2

Zimmerman, B. J. (1998). Academic studying and the development of personal skill: a self-regulatory perspective. Educational Psychologist, 33(2-3), 73-86. https:// doi.org/10.1080/00461520.1998.9653292 